\title{
Directed forgetting in rats: Evidence for active memory control?
}

\section{Chiaki Tanaka $^{1} \cdot$ Hayato Yahagi $^{1,2} \cdot$ Tohru Taniuchi $^{1}$}

Published online: 9 July 2019

(C) The Psychonomic Society, Inc. 2019

\begin{abstract}
Directed forgetting in rats, to elucidate active control of memory rehearsal processes while controlling for nonmemorial artifacts, was examined using an eight-arm radial maze. To-be-remembered and to-be-forgotten items were presented at different arms in the same trial. A trial consisted of learning and test phases. Rats needed to remember win or loss of a food pellet presented in the middle of the arms, signaling presence or absence of a large reward there in the subsequent test phase. Two other qualitatively different foods placed at the end of the arms served as remember $(\mathrm{R})$ or forget $(\mathrm{F})$ cues, signaling whether those arms would be presented in the test phase. Compared with the normal test, rats' performance deteriorated significantly if the arms previously marked by F-cues in the preceding learning phase were actually used in the test phase, showing reliable directed forgetting in rats. Rats were also tested in a condition in which F-cues were not presented at all, and thus rats had to remember all the arms. Although positive evidence of reduction of memory load in working memory by utilizing F-cues was not demonstrated, analysis of individual data suggested that utilization of R-cues and F-cues interfered with the main task of remembering win/lose information.
\end{abstract}

Keywords Rats $\cdot$ Directed forgetting $\cdot$ Radial maze $\cdot$ Working memory $\cdot$ Memory load $\cdot$ Active memory control

Humans have the ability to actively control rehearsal processes in working memory. This enables us to actively process our perceived world. For example, we try to retain important information in our working memory by selectively rehearsing the information. Such active control of the rehearsal ability must be adaptive in order to efficiently use the limited resources of working memory. This ability to actively control rehearsal has been investigated in humans using the directed forgetting paradigm, where a list of items includes cues for the items that will or will not be tested in a subsequent test. Subsequently, when all the items are tested, regardless of the remember $(\mathrm{R})$ or forget $(\mathrm{F})$ cues, poorer memory performance is shown for the F-items, directed by the F-cue, than for the Ritems, directed by the R-cue (e.g., Bjork, 1972; Golding,

This work was supported, in part, by JSPS KAKENHI Grant Numbers 21730589 and 24530913

Electronic supplementary material The online version of this article (https://doi.org/10.3758/s13420-019-00388-3) contains supplementary material, which is available to authorized users.

Tohru Taniuchi

tohruta@staff.kanazawa-u.ac.jp

1 Graduate School of Socio-Environmental Studies, Kanazawa University, 920-1192, Kakuma, Kanazawa, Ishikawa, Japan

2 College of Human and Social Sciences, School of Humanities, Kanazawa University, 920-1192, Kakuma, Kanazawa, Ishikawa, Japan
Long, \& MacLeod, 1994). Since it has been posited that directed forgetting reflects selective memory rehearsal for Ritems, but not for F-items, directed forgetting in another species could be evidence to suggest active control of the rehearsal process in that species (e.g., Roper \& Zentall, 1993).

The existence of working memory processes has been demonstrated in nonhuman animals, such as the monkey, pigeon, and rat, through delayed matching-to-sample (DMTS) or radial maze performance (e.g., Etkin \& D'Amato, 1969; Olton, 1978; Roberts, 1972). Therefore, can we conclude that animals that show reliable performance in DMTS or the radial maze have the ability to actively control working memory? No, because flexible use of information in working memory does not necessarily represent "active" control of memory processes. In order to support the hypothesis of active control of memory processes in nonhuman animals, directed forgetting has also been examined, primarily by modifying the DMTS task. In a typical DMTS task, a sample stimulus is presented, and, following a delay interval, two comparison stimuli are presented. A correct response to the comparison stimulus that matches the sample stimulus is rewarded. In a typical directed-forgetting version of DMTS, following the sample stimulus, an R-cue or an F-cue is presented during the delay interval. Comparison stimuli are then presented, and a response to the matching stimulus is rewarded for a trial in which the R-cue is presented. However, in a trial in which the F-cue is presented, the presentation of comparison stimuli 
is omitted, and the trial ends without testing. After sufficient acquisition training with R-trials and F-trials, probe test trials are occasionally inserted during training sessions. In probe trials, unlike normal training trials, comparison stimuli are presented following an F-cue, and memory of the sample stimulus is assessed. Poorer test performance has been observed in the probe test following an F-cue compared with the normal test for pigeons (Grant, 1981; Maki \& Hegvik, 1980; Maki, Olson, \& Rego, 1981, in their omission group; Parker \& Glover, 1987; Santi \& Savich, 1985; Stonebraker \& Rilling, 1981; Stonebraker, Rilling, \& Kendrick, 1981) and for monkeys (Roberts, Mazmanian, \& Kraemer, 1984, Experiment $3 b$ ). This type of directed-forgetting methodology is called an "omission procedure" because the memory test is omitted after the F-cue on the training trials. In another directed-forgetting procedure called the "substitution procedure," following the F-cue, instead of presenting comparison stimuli, a response to other stimuli is required in order to get a food reward on the training trials. In this procedure, reward opportunities are possible in the F-trials as well as the R-trials. Directed forgetting has been reported in the substitution procedure using pigeons (Grant, 1981; Grant \& Barnet, 1991; Schwartz, 1986) and rats (Grant, 1982). Based on these findings, it has been suggested that these animals, like humans, might have the ability to actively control rehearsal in working memory.

Roper and Zentall (1993) reviewed the initial directedforgetting studies in nonhuman animals and concluded that the data obtained through the omission and substitution procedures did not necessarily represent active control of rehearsal in nonhuman animals and could be interpreted as nonmemorial artifacts. For example, the F-cue in the omission procedure not only signals the omission of the sample memory test but also the omission of a reward opportunity. Thus, the F-cue could become a conditional stimulus that elicits conditional frustration. Such conditional frustration, if elicited by the F-cue, could interfere with test performance in the probe test. Also, as the F-cue signals the absence of a memory test with comparison stimuli, inattention to the test stimuli or a startle response caused by an "unpredicted" presentation of the test stimuli might interfere with test performance in the probe test. Some of these possible adverse effects of nonmemorial artifacts in the omission procedure were confirmed experimentally (Grant \& Soldat, 1995; Kaiser, Sherburne , \& Zentall, 1997; Zentall, Roper, \& Sherburne, 1995).

However, in the substitution procedure, a task that does not require sample memory is given instead of comparison stimuli. Therefore, unlike the omission procedure, a reward opportunity and attention to stimuli following the F-cue appear to be possible in the substitution procedure. However, Roper and Zentall (1993) also pointed out that the incompatibility between responses in a memory test with comparison stimuli following an F-cue in the probe test and a substitution response following an F-cue in the normal test could interfere with test performance in the probe test. For example, Grant (1981) examined directed forgetting in successive DMTS tasks with a single comparison stimulus presented following an Rcue (go/no-go discrimination), while a nondiscriminative substitution response was always required for a single constant visual stimulus following an F-cue. Pigeons might learn to respond to the stimulus following an F-cue indiscriminately, and thus they showed poor performance in the probe trials for which differential responses were required to the comparison stimuli following the F-cue. Therefore, demonstration of directed forgetting as a behavioral phenomenon does not necessarily imply an underlying active control of working memory processes. Possible nonmemorial artifacts must be eliminated carefully to demonstrate active control of memory processes in nonhuman animals.

Roper and Zentall (1993) also noted another important difference between directed forgetting tasks in human and nonhuman animals. In a typical human directed forgetting task, multiple R-items and F-items are presented in the same list. Thus, nonrehearsal of F-items could benefit the processing of R-items by reallocating limited working memory resources from F-items to R-items. However, in a typical directedforgetting experiment using DMTS in nonhuman animals, only one sample stimulus is presented, and testing memory of the sample stimulus depends on R-cues or F-cues. Therefore, nonrehearsal of a sample memory following an F-cue would not contribute to the retention of R-items.

Only a few studies have reported on directed forgetting in pigeons (Grant \& Soldat, 1995; Roper, Kaiser, \& Zentall, 1995) and rhesus monkeys (Tu \& Hampton, 2014) with adequate control for the nonmemorial artifacts pointed out by Roper and Zentall (1993). Notably, Roper et al. (1995; see also Kaiser et al., 1997) described directed forgetting in pigeons using a procedure that allowed for reallocation of memory resources from an F-item to an R-item by presenting an Ritem and one F-item in the same trial. In the R-trials, following an R-cue, pigeons were presented with comparison stimuli and were required to respond to a stimulus matching the sample. In contrast, in the F-trials of the task, either of two different $\mathrm{F}$-cues was presented, and pigeons were required to respond to comparison stimuli of either vertical or horizontal lines, depending on the F-cues, but not the sample stimulus. Hence, in the F-trials, reallocating memory resources from the sample stimulus (the F-item in the trial) to the F-cue stimulus (R-item in the trial) could contribute to good performance.

Therefore, true directed forgetting has been shown for avians and primates with appropriate control of nonmemorial artifacts, as per Roper and Zentall (1993). Although some studies have shown directed forgetting with an omission procedure (Grant, 1982, no-test group; Miller \& Armus, 1999) or a substitution procedure (Grant, 1982, no-choice group) in 
rats, appropriate control of nonmemorial artifacts has not yet been demonstrated. Additionally, an experimental procedure allowing for reallocation of memory resources from $\mathrm{F}$-items to $\mathrm{R}$-items has not been tested in rats. Comparisons of directed forgetting among various species, in addition to avians and primates, is needed in order to clarify the origins of active control of memory processes.

The purpose of the present study was to examine directed forgetting in rats using a procedure that allows for reallocation of memory resources from F-items to R-items with appropriate control of nonmemorial artifacts. In Experiment 1, rats were trained and tested in a directed forgetting task in which $\mathrm{R}$-items and $\mathrm{F}$-items were presented in the same trials. Experiment 2 examined the replicability of the findings in Experiment 1. Experiment 3 investigated whether rats do reallocate memory resources from F-items to R-items.

\section{Experiment 1}

In Experiment 1, rats were tested in a directed forgetting task using an elevated eight-arm radial maze (see Fig. 1). Each trial consisted of a learning phase, a delay interval, and a test phase (see Fig. 2). Although a standard radial maze has only one food cup at the end of each arm, the radial maze in the present study had a food cup at both the midpoint and end of each maze arm. To-be-remembered memory items were the win or loss of a food pellet in the middle cup of the eight arms. Additionally, two qualitatively different foods, placed in the end cups, acted as R-cues and F-cues to alert rats as to whether the arm would be used in the subsequent test phase. Reportedly, rats are sensitive to differences in the quality or quantity of food rewards and use them as signals to discriminate subsequent events (e.g., Capaldi \& Miller, 1988; Phelps $\&$ Roberts, 1991). In the learning phase, where the first word represents win or loss of a food pellet in the middle cup and the second character represents the R-cues or F-cues presented in the end cup, one lose-R arm (i.e., no food pellet in the middle cup and R-cue in the end cup), two lose-F arms, and five win-R arms were presented (see Table 1). In the learning phase, rats were allowed to enter the eight arms one by one in a predetermined random order by opening the doors one by one. Since the doors of the visited arms were closed immediately after a visit, rats could not enter those arms again, and the learning phase always had eight choices. For example (see Fig. 1), after a rat entered an arm from the central platform, it had to climb over a barrier first, then encountered the win or loss of a food pellet in the middle cup and, at the end of the arm, received a R-cue or F-cue (qualitatively different foods) in the end cup. After returning to the central platform, the rat was allowed to enter a next designated arm in turn until it visited all eight arms.

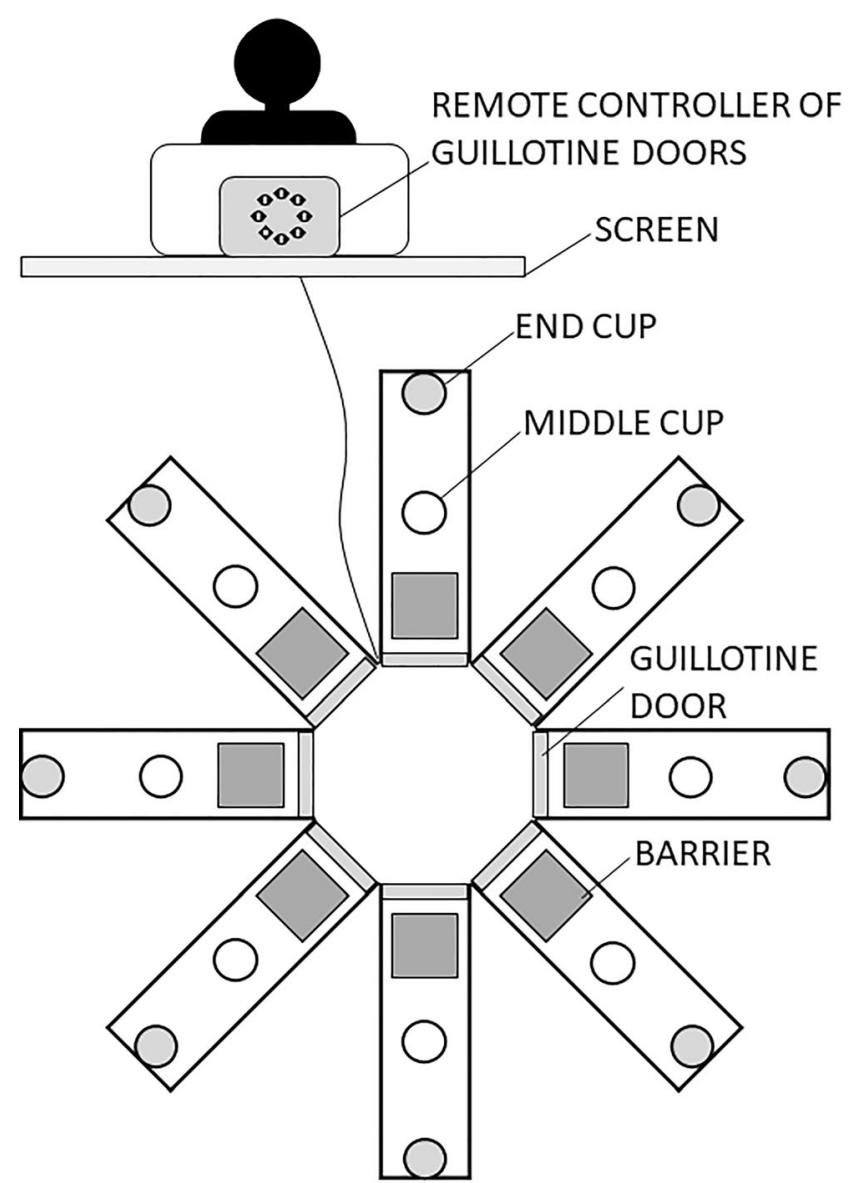

Fig. 1 Schematic of the radial maze in Experiment 1. Food cups were set at the middle and end of each arm. An obstruction barrier, 12-cm high, was placed at the entrance to each arm in order to increase response cost for entering the arm. Doors of the arms were controlled electrically by the experimenter behind a semitransparent screen

There were two reasons that we used the forced-choice procedure in the learning phase. First, in our pilot study, in which a free-choice procedure was used in the learning phase, rats came to circle the maze arms continuously in one direction (Roberts \& Dale, 1981). Such a stereotypic response pattern appeared to interfere with test performance, and rats did not demonstrate reliable acquisition of the task. By presenting the arms in a random order in the learning phase, we expected this would help prevent the development of a stereotypic response pattern. In addition, if we allowed reentry to arms in the learning phase, rats might find no food in the middle and end cups in the second or later choice of the arm. Thus, memories of those nonreward events in the succeeding choices would interfere with those of the first entry. To prevent rats from experiencing such extraneous reward events in succeeding choices, we adopted the forced-choice procedure.

In the test phase, following a 3-min delay interval, the two lose-F arms of the prior learning phase were excluded from the test by keeping their entrance doors closed during the test phase. Ten food pellets were placed in the middle cup of the 


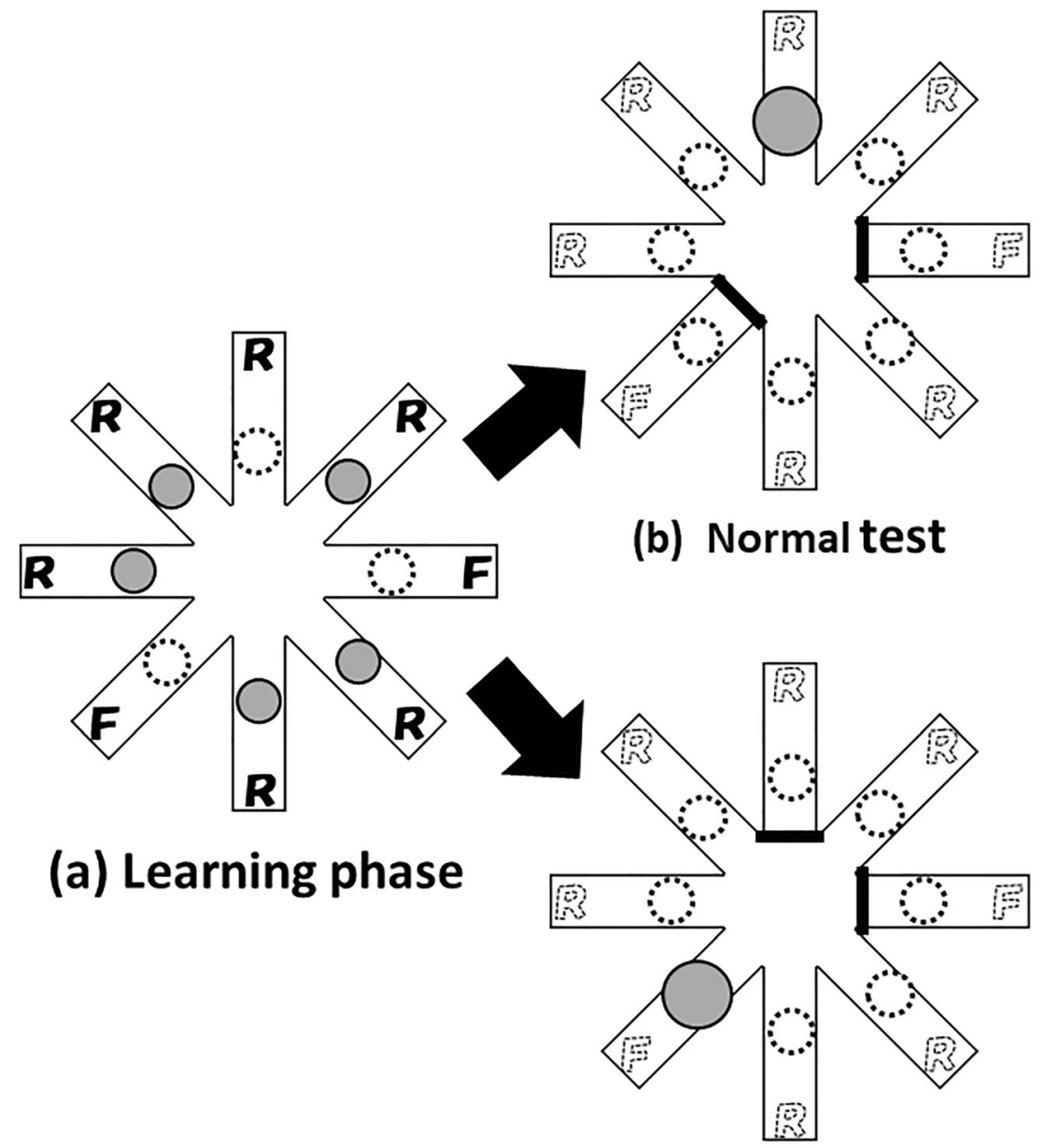

\section{(c) Probe test}

Fig. 2 An overview of procedures in Experiments 1 and 2. A training trial consisted of a learning phase (a), a delay interval of 3 min., and a normal test phase (b). During the probe test period, following acquisition training, a probe test (c) was inserted instead of the normal test in one out of six trials. Filled gray circles in the middle of the arms represents one food pellet in the learning phase (a) or 10 (Experiment 1) or 20 (Experiment 2) food pellets in the test phase (b and $\mathbf{c}$ ). Broken circles

lose- $\mathrm{R}$ arm from the learning phase, and no reward was placed in the other middle and end cups (win-shift/lose-stay task). represent a nonreward in the middle food cups. Solid $\mathrm{R}$ and $\mathrm{F}$ at the end of the arms represent two qualitatively different foods presented as remember-cues (R-cues) and forget-cues (F-cues) in the end cups, respectively. Broken $\mathrm{R}$ and $\mathrm{F}$ represent $\mathrm{R}$-cues and $\mathrm{F}$-cues presented in the prior learning phase. Bold lines at the entrances of the arms represent closed doors in the test phase (b and $\mathbf{c})$

That is, if a rat found a food pellet in the middle cup of an arm in the learning phase, it would not be rewarded for

Table 1 Outline of procedures for the training and probe test trials in Experiments 1 and 2

\begin{tabular}{lll}
\hline & Learning phase & Test phase \\
\hline Training trial & One lose-R arm & Correct arm \\
& Two lose-F arms & Excluded from testing \\
& Five win-R arms & Incorrect arms \\
Probe test trial & One lose-R arm & Excluded from testing \\
& Two lose-F arms & One of the arms was correct and the other was excluded from testing \\
& Five win-R arms & Incorrect arms \\
\hline
\end{tabular}

Note. Win and lose represent the presence or absence of a $45-\mathrm{mg}$ food pellet in the middle cup in the learning phase, respectively. $\mathrm{R}$ and $\mathrm{F}$ stand for presentation of remember or forget cues (two qualitatively different foods) in the end cup in the learning phase, respectively. In the test phase, 10 (Experiment 1) or 20 (Experiment 2) food pellets were presented in the middle cup of the correct arm, and no reward was placed in the incorrect arms. Doors of two out of eight arms remained closed and excluded from the test in both trials 
entering that arm in the test phase. Conversely, if a rat found no food in the middle cup of an arm in the learning phase, it could predict a large reward in that arm in the test phase. Thirdly, if the rat encountered an F-cue in the end cup of the arm, it could predict that the arm would be excluded in the test phase.

Hence, although rats had to remember win or loss of a food pellet in the middle cup in the learning phase in order to choose a correct arm in the test phase of the trial, if rats could utilize R-cues and F-cues in the learning phase to predict which arms would be presented in the subsequent test phase, they could exclude the two lose-F arms from working memory since those two arms would be excluded from the test. After acquisition training, one probe trial was inserted for each five normal training trials. In the probe trials, contrary to the directions implied by the R-cues and F-cues in the learning phase, a lose-F arm was presented in the test phase instead of a lose- $\mathrm{R}$ arm as the correct arm. Poorer test performance in the probe trials than in the normal training trials was to be expected if rats could actively exclude F-arms from working memory by utilizing R-cues and F-cues.

\section{Method}

\section{Subjects}

The subjects were 10 experimentally naïve, male Wistar rats, approximately 120 days old and weighing $408.0 \mathrm{~g}$ on average at the start of the experiment. Rats were given $16 \mathrm{~g}$ of food daily except for experimental rewards. These rats were acquired from Kiwa Laboratory Animals Co., Ltd., and were cared for and used according to guidelines approved by Kanazawa University Animal Experimentation Regulations.

\section{Apparatus}

An eight-arm radial maze, elevated $60 \mathrm{~cm}$ above room floor level (see Fig.1), was used. The maze floor was constructed of plywood and painted flat gray. The octagonal central platform was $34 \mathrm{~cm}$ in diameter and surrounded by transparent walls and guillotine doors, which were controlled electrically by a remote control system and rotary solenoids. The radial maze arms were 9-cm wide and 76-cm long, with gray PVC walls 2$\mathrm{cm}$ high. Adjacent arms were separated by $45^{\circ}$ angles, and two food cups were set on each arm. One food cup, $4 \mathrm{~cm}$ in diameter, was located in the middle of each arm, and the other food cup, $3.5 \mathrm{~cm}$ in diameter, was buried at the end of each arm. Since it has been reported that increasing the response cost of visiting arms contributed to better performance in radial maze tests (e.g., Brown, 1990; Brown \& LesniakKarpiak, 1993), an obstruction (6-cm long, 6-cm wide, and $12-\mathrm{cm}$ high), made of two bricks, was placed $10 \mathrm{~cm}$ from the entrance to an arm in order to increase the response cost of entering the arm. A transparent resin cage (40-cm long, 26-cm wide, and $20-\mathrm{cm}$ high) with bedding materials was used to contain the rats during the delay interval. Forty-five mg of food pellets, $30 \mathrm{mg}$ of puffed sweetened rice, and $150 \mathrm{mg}$ of chocolate chips were used for food rewards and R-cues or Fcues, as described below. The experimental room was $5.0-\mathrm{m}$ long, 3.5-m wide and contained a number of distinct extramaze cues, such as a table with two operant boxes, black curtains, a metal shelf, a ceramic sink, and two doors. A semitransparent brown PVC board prevented the rats from seeing the experimenter manipulate the remote controls of the maze.

\section{Procedure}

Pretraining During the first 8 days, rats were handled for 1 min each day, and on Days 4-8, five food pellets, five grains of sweetened puffed rice, and five chocolate chips were placed in their home cages to familiarize the rats with these foods. Exploration of the maze was allowed from Day 9 for 4 days, and the rats could explore the maze for 15 minutes each day individually. All the doors were open, and rats could eat a food pellet from each of the middle and end cups. Training in entering the radial maze arms began on Day 13 and lasted for 17 days. The arms were serially opened, one by one, in random order, and rats could enter to eat a food pellet from both the middle and the end cup. An obstacle was erected $10 \mathrm{~cm}$ beyond the entrance to an arm in order to increase the response cost. Initially, there was no obstacle, then, a 5-cm long, $5-\mathrm{cm}$ wide, and $1.5-\mathrm{cm}$ high metal board was put in place. Next, two metal boards were stacked to make a $3-\mathrm{cm}$ high obstacle. This was replaced by a 6-cm long, 6-cm wide, and 6-cm high brick, and ultimately, two bricks were stacked up to make the $12-\mathrm{cm}$ high obstacle used in the experiment.

Acquisition training Acquisition training began on Day 30 and lasted for 36 days. The three steps in the acquisition trials were the learning phase, delay interval, and test phase. In the learning phase, using the radial maze (see Fig. 2a), one arm had no food pellet in the middle cup, and the R-cue food in the end cup (lose-R), two arms had no food pellet in the middle cup and the F-cue food in the end cup (lose-F), and remaining five arms had a food pellet in the middle cup and the R-cue food in the end cup (win-R). R-cues and F-cues were counterbalanced between the three grains of puffed sweetened rice and the chocolate chips across subjects. A rat was placed in the central platform with all doors closed and about $5 \mathrm{~s}$. later, the doors were opened one by one in a predetermined random order. Rats were allowed to enter the arms until all eight arms had been visited. Since the doors of the visited arms were closed after a visit, rats could not enter those arms again, and the learning phase always had eight choices. Assignment of one lose-R, two lose-F, and five win- $\mathrm{R}$ arms was changed randomly trial by trial. When the rat had visited all eight arms and returned to the central 
platform, it was moved to a waiting cage by the experimenter and kept there for a 3-min delay interval. After the delay interval, the test phase was conducted. For the normal test phase, the middle food cup of the lose- $\mathrm{R}$ arm in the prior learning phase was baited with 10 food pellets (correct arm in the test phase), and no food was put in other middle and end cups (see Fig. 2b). The rat was placed in the central platform, and about $5 \mathrm{~s}$ later, the six doors of the single lose- $\mathrm{R}$ arm and five win- $\mathrm{R}$ arms from the prior learning phase were opened simultaneously. Doors of two lose-F arms from the learning phase remained closed and excluded from the test. Since the doors of the visited arms were closed immediately after a visit, rats could not enter those arms again, and the test phase always finished within six choices. First, rats were allowed to choose arms freely until they found the correct arm, but then the number of arms they could visit was gradually restricted: six visits for the first 10 days, three visits for next 10 days, and two visits for the remaining 16 days. This gradual restriction of the number of arms allowed to visit in the test phase was introduced because in our pilot study, without such restriction, most of rats did not learn the task. That is, rats continued to choose arms seemingly at random, without using their memory of the learning phase, until they found a large reward in the test phase. It was expected that restricting the number of the arms allowed to visit would help make rats use their memory of the learning phase and learn the task. After consumption of the food reward of 10 food pellets in the correct arm, the rat was returned to its home cage. Other aspects of the procedure were identical to the learning phase. Two trials were conducted per day with a 60-80-min intertrial interval. Assignment of the one lose-R, two lose-F, and five win- $\mathrm{R}$ arms was changed randomly and independently between the two daily trials.

Probe test After completing 36 days of acquisition training, probe tests were conducted for 18 days. One probe trial was given in each block of 3 days, and six probe trials were conducted in total for each rat. The remaining five trials in each block of 3 days were normal training trials as used in acquisition training. In the probe test, in contradiction to the R-cuing and F-cuing of the learning phase, one of the two lose-F arms was randomly selected and presented in the test phase (see Fig. 2c) instead of the lose- $\mathrm{R}$ arm. The number of visits was restricted to two arms in both the probe and the normal training trials. Other aspects of the procedure in the probe trials were identical to those in acquisition training. The order of the probe trial, as the first or second trial in each daily session, alternated with each subject and was counterbalanced across subjects.

\section{Results}

During preliminary training, four out of 10 rats were excluded from the experiment because they had difficulty in smoothly climbing over the obstacle placed at the entrance to each arm, while the other six rats participated in the remainder of the experiment. During pretraining, all rats learned to go to the middle cup first and then to the end cup. No exceptional behavior was observed by the experimenter during acquisition training and the probe test period.

Figure 3 shows the mean rank of correct choices during acquisition training in blocks of four trials. Since the number of arms that could be visited in the test phase was gradually restricted from six to two, in order to evaluate the rats' performance using an identical index throughout acquisition training, we assigned a rank of 5.0 (average of fourth, fifth, and sixth) if rats failed to choose the correct arm when restricted to three arms and a rank of 4.5 (average of third, fourth, fifth, and sixth) if rats failed to choose the correct arm when restricted to two arms. The broken line represents the chance level (3.5). Although the rats' initial performance was around the chance level, it improved gradually and reached 2.21 in the last block. A one-factor, repeated-measures ANOVA of block (18 total) showed that the main effect of block was not significant, $F(17$, $85)=.884, p=.593, \eta_{\mathrm{p}}{ }^{2}=.150$. However, the upper limits of the $95 \%$ confidence interval were reliably better than the chance level of 3.5 in Blocks 5, 6, 9, and 14 through 18. Since better-than-chance performance was shown primarily in the later blocks, it suggests that rats learned to choose the correct arm at better-than-chance levels through acquisition training.

The top panel of Fig. 4 shows the mean rank of correct responses during the probe test period. At $95 \%$ confidence interval analysis, the rats' performance was reliably better than the chance level of 3.5 during the normal training trials (1.675 $<\mu<3.125$ ), whereas it was not significant for the probe trials $(2.928<\mu<4.072)$. Paired $t$ tests showed that the rats' test performance was poorer in the probe test than in the normal test, $t(5)=2.82, p=.019, r=.78$, one-tailed. The bottom panel of Fig. 4 shows the percentage correct of their first choice during the probe test period. At the $95 \%$ confidence interval analysis, rats' performance was reliably better than chance (16.67\%) for the normal training trials $(20.108<\mu<$ 61.002), whereas it was not significant for the probe trials $(-2.493<\mu<35.827)$. Paired $t$ tests showed that the rats' test performance was poorer in the probe test than in the normal test, $t(5)=3.196, p=.012, r=.82$, one-tailed. These results show that the rats' performance consistently deteriorated if the correct arm used in the test phase of probe trial had previously been signaled incorrectly by the F-cue in the prior learning phase.

\section{Discussion}

Experiment 1 examined the rats' performance in a directed forgetting task in which R-items and F-items were presented in the same trial, allowing reallocation of memory resources 


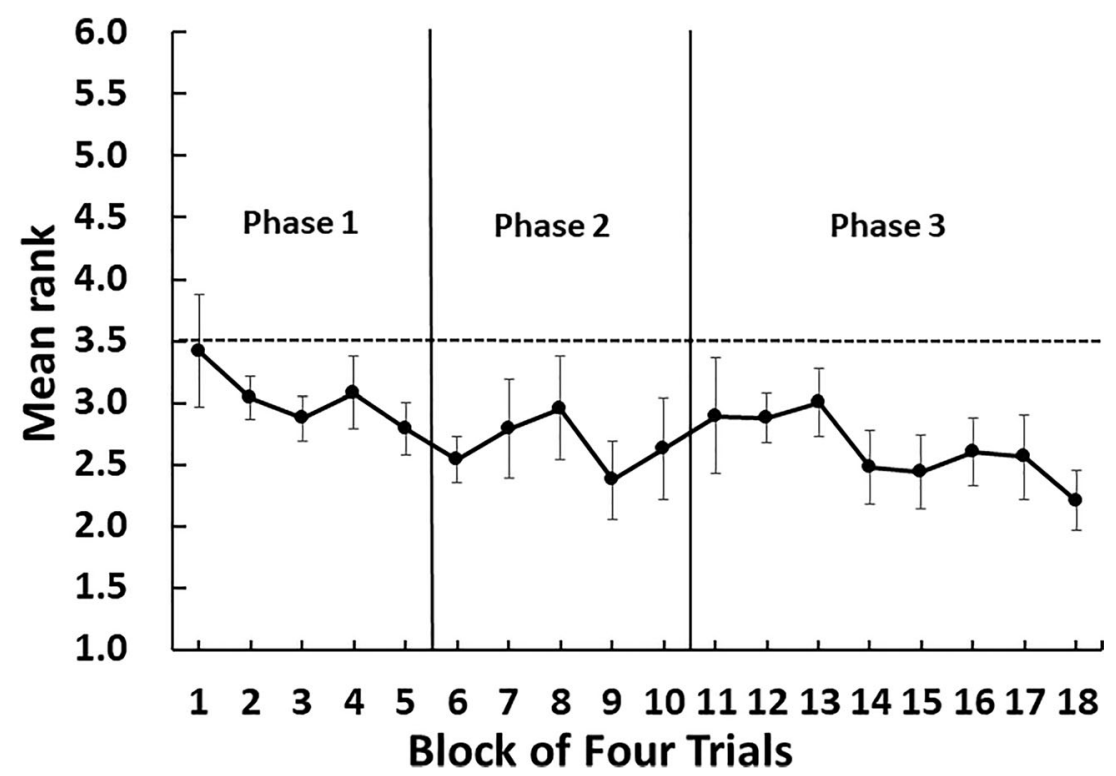

Fig. 3 Mean rank of correct choice during acquisition training in Experiment 1. Vertical lines represent shifts in condition for allowed number of choices. Rats were allowed six (Blocks 1-5), three (Blocks

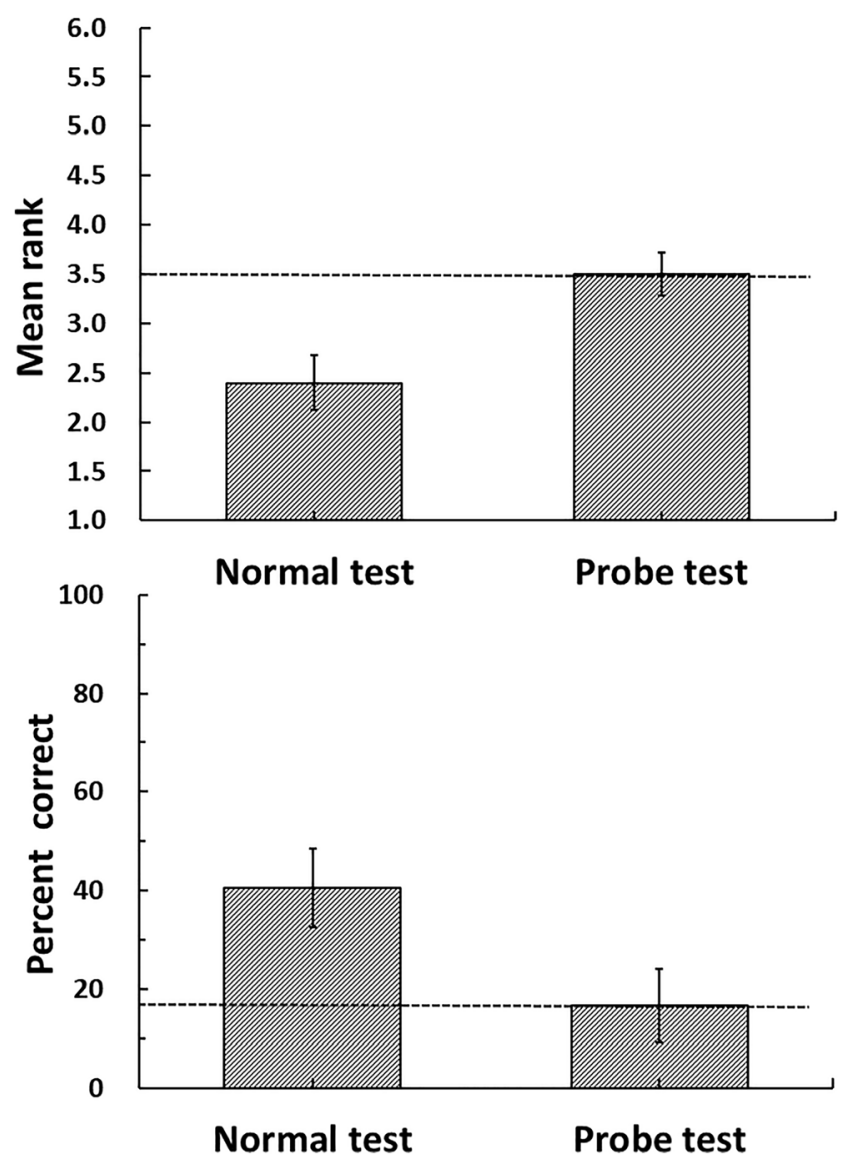

Fig. 4 Top panel: Mean rank of correct choice in the normal and the probe tests during the probe test period in Experiment 1. The broken line represents chance level (3.5). Bottom panel: Percentage correct of first choice in the normal and the probe tests during the probe test period. The broken line represents chance level (16.67\%). Error bars represent standard error of the mean
6-10), or two (Block 11-18) choices in the test phase. Error bars represent standard error of the mean

from F-items to R-items. The rats' test performance significantly deteriorated in the probe trials in which the correct arm had been signaled incorrectly by an F-cue in the prior learning phase. These results demonstrate that the task used in Experiment 1 may be an effective means to elicit directed forgetting in rats.

Previous research in rats, which used an omission or substitution procedure, could not eliminate the possibility of nonmemorial artifacts, such as interference of conditional frustration elicited by an F-cue, lack of attention to test stimuli, interference from surprise by presenting test stimuli following an F-cue, or incompatibility between response to test stimuli and substitution response (Grant, 1982; Miller \& Armus, 1999). Unlike previous research with rats, R-items and Fitems were presented in the same trial in this study. The Fcue in the learning phase signaled only "exclusion of F-items in the subsequent test" and did not signal any omission of reward opportunity in this study. Therefore, it is very unlikely that conditional frustration, as a result of pairing the F-cue with an omission of reward opportunity, developed in Experiment 1. Also, rats were required to choose the one correct arm out of six arms in the test phase in both the normal training trials and the probe trials. Therefore, attention to test arms should have been equal in both the normal training trials and the probe trials. Since the response required in the test phase of the normal training trials and the probe trials was identical, there was not any incompatibility between the required response in the normal training trials and in the probe trials. Finally, in the directed forgetting procedure used in Experiment 1, six R-cues and two F-cues were always presented in the learning phase of both normal training trials and probe trials. Thus, the possibility of interference from surprise 
in the probe test's omission procedure would not occur selectively in the test phase of the probe trials in Experiment 1. Hence, the reliable directed forgetting effects in Experiment 1 cannot be explained in terms of possible nonmemorial artifacts, as pointed out by Roper and Zentall (1993). Therefore, the findings in Experiment 1 strongly suggest true directed forgetting in rats and their active control of rehearsal of Ritems and F-items presented in the same trial.

We assigned the mean rank of remaining arms for trials in which rats could not respond to the correct arm within the number of arms allowed to visit. Such substitution assumes that the rat had no memory of the correct arm and it would choose the remaining arms at random. However, if rats failed to respond to the correct arm, they still might have some memory of the correct arm. That is, our analysis might underestimate rats' test performance. However, since this analysis possibly underestimates, but not overestimates, rats' performance on the error trials, our data that showed rats' performance were reliably improved from the initial phase to the final phase must logically be more conservative than reality. That is, rats' performance was significantly better in the later phases of acquisition (with possibly greater underestimation of rats' memory) than that in the initial phases (with possibly less underestimation of rats' memory).

Additionally, the restriction of the number of arms allowed to visit should also not affect our conclusion in the comparison between performance in the normal and the probe tests because the allowed arm entries were both two in the normal and probe tests. In this regard, our conclusion about reliable acquisition of the task and significant directed forgetting effects must be still valid despite the possible underestimation of rats' memory performance by the substituted rank for error trials.

\section{Experiment 2}

In Experiment 1, rats showed reliable directed forgetting effects in a radial maze task in which R-items and F-items were presented in the same trial. Directed forgetting in rats has been investigated mainly through the DMTS task using a T-maze (Grant, 1982) or an operant chamber (Miller \& Armus, 1999). In this regard, the directed forgetting task and apparatus used in Experiment 1 was considerably different from those used in previous research with rats, and there are no other similar results to support our findings. Recently, the importance of replication of findings in psychology, especially with greater statistical power, has been emphasized (e.g., Maxwell, Lau, \& Howard, 2015). Experiment 2 attempted to examine the replicability of the findings of Experiment 1 with a larger number of subject animals. Considering the possibility of proactive interference in spatial memory in the radial maze task (Cohen, Reid, \& Chew, 1994; Cohen, Sturdy, \& Hicks,
1996; Roberts \& Dale, 1981), the number of daily trials was reduced from two in Experiment 1 to one in Experiment 2. The obstruction made of bricks placed in the arms in Experiment 1 was not used in Experiment 2 because some rats had difficulty climbing smoothly over the obstacles in Experiment 1. The food reward for a correct response in the test phase was increased from 10 pellets in Experiment 1 to 20 pellets in Experiment 2. Different schedules to reduce the number of arms rats were allowed to visit in the test phase during acquisition training were also used for Experiment 2 because the schedule was determined in accordance with the progress of acquisition. Other aspects of the experimental procedure in Experiment 2 were similar to those of Experiment 1.

\section{Method}

\section{Subjects}

The subjects were 10 experimentally naïve, male Wistar rats, approximately 90 days old and weighing $250.3 \mathrm{~g}$, on average, at the start of Experiment 2. Rats were given $16 \mathrm{~g}$ of food daily except for experimental rewards. The rats were acquired from Sankyo Labo Service Corp., Inc., and were cared for and used according to guidelines approved by Kanazawa University Animal Experimentation Regulations.

\section{Apparatus}

The brick obstacles placed at entrances to the maze arms in Experiment 1 were not used in Experiment 2. All other aspects of the apparatus were identical to those of Experiments 1.

\section{Procedure}

Pretraining During the first 11 days, rats were handled for $3 \mathrm{~min}$ daily, and five food pellets, five grains of sweetened puffed rice, and five chocolate chips were placed in their home cages for familiarization. Exploration of the apparatus was allowed from Day 12 for 4 days. Each rat could explore the maze for $15 \mathrm{~min}$ daily. All eight doors of the radial maze were open, and rats could eat a food pellet from the middle cup and a chocolate chip and three grains of puffed sweetened rice from the end cup. Training in entering the radial maze arms began on Day 16 and lasted for 18 days. The arms were serially opened, one by one, in random order, and rats could enter to eat a food pellet from the middle cup and a chocolate chip and three grains of puffed sweetened rice from the end cup. Other aspects of pretraining were identical to those of Experiment 1.

Acquisition training Acquisition training began on Day 34 and lasted for 68 days. As in Experiment 1, an acquisition trial consisted of a learning phase, a delay interval, and a test phase. 
In the learning phase, one lose- $\mathrm{R}$ arm, two lose-F arms, and five win-R arms were presented (see Table 1). The doors were opened serially, one-by-one, in a random order without replacement. Since the doors of the visited arms were closed after a visit, rats could not enter those arms again. In the test phase following the 3-min delay interval, the middle food cup of the lose- $\mathrm{R}$ arm in the learning phase was baited with 20 food pellets (correct arm in the test phase), and no food was placed in the remaining middle and end cups. The doors of the two lose-F arms in the learning phase remained closed and excluded from the test. The number of allowable visits to arms was gradually restricted, depending on actual acquisition progress: six visits for the first 12 days, five visits for next 8 days, four visits for next 12 days, and three visits for the last 36 days. A single trial was conducted during each daily session. Other aspects of the procedure were identical to those of Experiment 1.

Probe test After the completion of acquisition training, a probe test period was conducted for 12 days. One probe trial was given for each block of 4 days, and a total of three probe trials were carried out on each rat. The remaining three trials in each block of 4 days were normal training trials, just as in the acquisition-training period. A probe trial was conducted on the second or third day of the 4-day block and was counterbalanced across subjects. In the test phase of the probe trial, one of the two lose-F arms was randomly selected to replace the lose-R arm, and the number of visits was restricted to three arms. All other aspects of the procedure in the probe trials were identical to those of acquisition training.

\section{Results}

Figure 5 shows the mean rank of correct choices during acquisition training in blocks of four trials. Since the number of allowable visits to arms in the test phase was gradually restricted from six to three, in order to analyze the rats' performance using an identical index throughout acquisition training, a rank of 5.5 (average of fifth and sixth) and 5.0 (average of fourth, fifth, and sixth) was given if rats failed to choose the correct arm when restricted to four and three arms, respectively. A broken line represents the chance level of 3.5. Although rats' initial performance was around chance, it improved gradually and became better than chance, especially in the final blocks. A one-way repeated-measures ANOVA of Block 17 showed significant main effect of block, $F(16,144)=2.110, p$ $=.011, \eta_{\mathrm{p}}^{2}=.19$. Pairwise comparison by $t$ test with adjusted significance level by Ryan's method revealed that rats' performance was significantly better in Block 16 than in Blocks 1, 4, 5 , and 9. Moreover, the upper limits of the $95 \%$ confidence intervals were reliably better than the chance level of 3.5 in Blocks 15, 16, and 17. These results suggest that rats learned to reliably choose the correct arm better than chance due to acquisition training.

The top panel of Fig. 6 shows the mean rank of correct response in the normal training trials and the probe trials during the probe test period. According to a $95 \%$ confidence interval analysis, rats' performance was reliably better than the chance level of 3.5 for the normal training trials $(1.675<$ $\mu<3.125$ ), whereas it was not significant for the probe trials $(2.928<\mu<4.072)$. Also, paired $t$ tests showed that rats' test performance was poorer in the probe test than in the normal test, $t(9)=2.156, p=.030, r=.58$, one-tailed. The bottom panel of Fig. 6 shows the percentage correct of the first choice during the probe test period. According to a $95 \%$ confidence interval analysis, rats' performance was reliably better than the chance level of $16.67 \%$ for the normal training trials $(18.122<$ $\mu<41.878$ ), whereas it was not significant in the probe trials $(-.194<\mu<33.528)$. Paired $t$ tests showed that rats' test performance was poorer in the probe test than in the normal test, $t(9)=1.964, p=.041, r=.55$, one-tailed. These results were consistent with those of Experiment 1, showing that rats' performance reliably deteriorated if the correct arm in the test phase had been signaled incorrectly by an F-cue in the prior learning phase.

\section{Discussion}

As in Experiment 1, rats reliably learned to choose the correct arm during acquisition training in Experiment 2. Although the main effect of the training block was not significant in Experiment 1, it reached a level of significance in Experiment 2. More importantly, the results in Experiment 1 , which showed poorer performance in probe trials rather than in the normal tests, were also replicated in Experiment 2. That is, rats' test performance deteriorated if the correct arm had been signaled incorrectly by an F-cue in the prior learning phase. These results suggest that the directed forgetting effect in the present paradigm, in which nonmemorial artifacts were controlled and the reallocation of memory resources from Fitems to R-items was allowed, has stable replicability and intersubject generality.

Speed of acquisition was somewhat slower in Experiment 2 than in Experiment 1. There were some differences in procedure between Experiments 1 and 2. Since the reduction of the number of daily trials was expected to reduce possible proactive interference between daily trials (Roberts \& Dale, 1981), that could not be a reason for slower acquisition in Experiment 2. Larger food rewards to correct responses in the test phase in Experiment 2 than in Experiment 1 was also expected to contribute to better test performance. In contrast, some research has reported that increasing the response cost of visiting arms by manipulating length or incline of the arms contributed to better performance in a radial maze (e.g., Brown, 1990; Brown \& Lesniak-Karpiak, 1993). Therefore, 


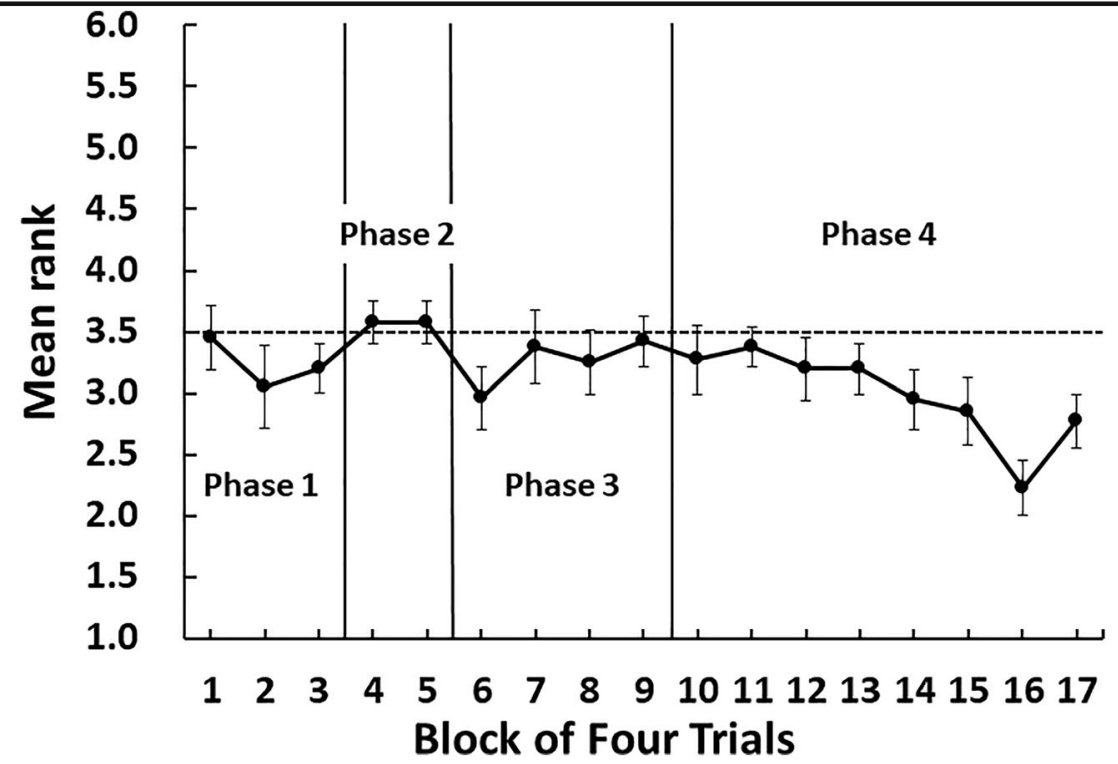

Fig. 5 Mean rank of correct choice during acquisition training in Experiment 2. Vertical lines represent shifts in condition for allowed number of choices. Rats were allowed six (Blocks 1-3), five (Blocks 4

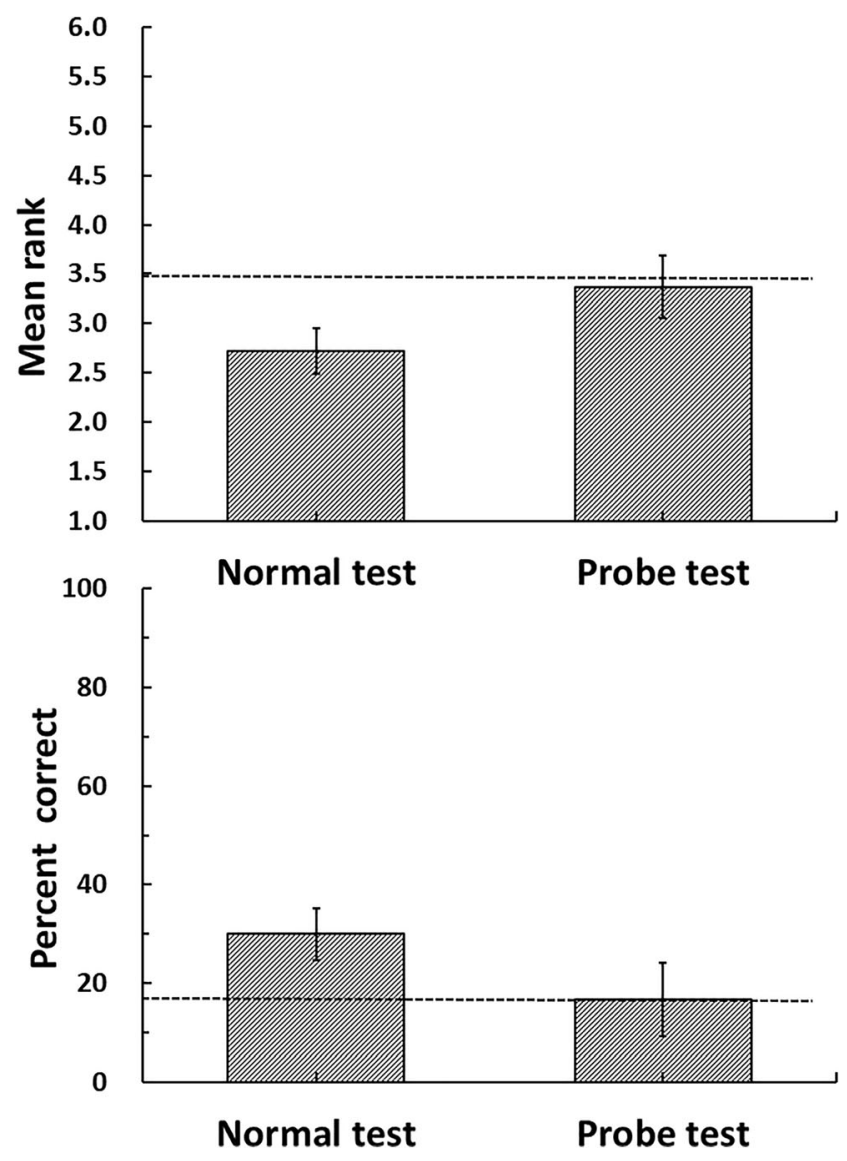

Fig. 6 Top panel: Mean rank of correct choice in the normal and probe tests during the probe test period in Experiment 2. The broken line represents chance level (3.5). Bottom panel: Percentage correct of first choice in the normal and the probe tests during the probe test period. The broken line represents chance level (16.67\%). Error bars represent standard error of the mean
5), four (Block 6-8), or three (Block 9-17) choices in the test phase. Error bars represent standard error of the mean

the difference in acquisition performance between Experiments 1 and 2 might be explained in terms of a difference in response cost to visit the arms. Although introducing such obstructions requires shaping training to climb over them, it might contribute to better performance in this relatively complex memory task in the radial maze.

\section{Experiment 3}

It appears that directed forgetting, using rats in a radial maze, may have been demonstrated in Experiment 1 and replicated in Experiment 2. These results suggest that rats have the ability to control their memory rehearsal actively. The purpose of Experiment 3 was to provide further evidence of the directed forgetting effect shown in Experiment 2, reflecting the reallocation of memory resources from F-items to R-items. Rats in Experiment 2 continued to be tested in Experiment 3, and performance in low-memory-load and high-memory-load conditions was compared, using a within-subjects design. The procedure in the low-memory-load condition was identical to that in the normal training trials of Experiment 2. That is, one lose- $\mathrm{R}$ arm, two lose-F arms, and five win- $\mathrm{R}$ arms were presented in the learning phase of the low-memory-load condition (see Table 2). In contrast, F-cues were not used in the high-memory-load condition. That is, three lose- $\mathrm{R}$ arms and five win- $R$ arms were presented in the learning phase of the high-memory-load condition. Since the correct arm in the test phase was selected randomly from three lose- $R$ arms in the learning phase, rats had to remember all these possible correct arms in the high-memory-load condition. Conversely, in the low-memory-load condition, rats could reallocate memory 
Table 2 Outline of procedures for the low-memory-load and the high-memory-load trials in Experiment 3

\begin{tabular}{lll}
\hline & Learning phase & Test phase \\
\hline Low-memory-load trial & One lose-R arm & Correct arm \\
& Two lose-F arms & Excluded from testing \\
& Five win-R arms & Incorrect arms \\
High-memory-load trial & Three lose-R arm & One of the arms was correct and the other two arms were excluded from testing \\
& Five win-R arms & Incorrect arms \\
\hline
\end{tabular}

Note. Doors of two out of eight arms remained closed and excluded from the test in both trials

resources for the two lose-F arms to the R-arms. Therefore, if rats used $\mathrm{R}$-cues and $\mathrm{F}$-cues and reallocated memory resources from the F-arms to the R-arms, better test performance would be expected in the low-memory-load condition than in the high-memory-load condition.

\section{Method}

\section{Subjects}

The 10 rats from Experiment 2 were used in Experiment 3. The treatment of the animals was identical to that of Experiment 2.

\section{Procedure}

Experiment 3 began the day after the completion of Experiment 2. As in Experiments 1 and 2, a trial consisted of a learning phase, a 3-min delay interval, and a test phase. Low-memory-load and high-memory-load conditions were assigned to the rats using a within-subjects design. In the low-memory-load trials (see Fig. 7-a1), one lose-R arm, two lose-F arms, and five win- $\mathrm{R}$ arms were presented in the learning phase, exactly as in the training trials of Experiment 2. In the high-memory-load trials (see Fig. 7-b1), F-cues were not used, and three lose- $\mathrm{R}$ arms and five win- $\mathrm{R}$ arms were presented in the learning phase. The test phase was conducted after a 3-min delay interval. In the test phase of low-memoryload trials (see Fig. 7-a2), the middle food cup of the lose-R arm in the learning phase was baited with 20 food pellets and no food was placed in remaining middle and end cups. The doors of the two lose-F arms remained closed and excluded from the test. In the test phase of the high-memory-load trials (see Fig. 7-b2), one of the three lose-R arms from the prior learning phase was selected randomly and its middle cup was baited with 20 food pellets. The doors of the other two lose- $\mathrm{R}$ arms remained closed and excluded from the test and the number of allowable visits to arms was restricted to three. Experiment 3 was conducted over 24 days. The highmemory-load trial was given on the second or third day (counterbalanced among subjects) in a 4-day block, and the other three trials tested the low-memory-load condition. Thus, a total of 18 trials in the low-memory-load condition and six trials in the high-memory-load condition were conducted. Other aspects of the procedure were identical to those of the normal test trials in Experiment 2.

\section{Results}

The top panel of Fig. 8 shows the mean rank of correct choices in the low-memory-load and high-memory-load conditions. Since the number of arms visited in the test phase was restricted to three, a rank of 5.0 (average of fourth-sixth) was given if rats failed to choose the correct arm within the restriction of three visits. Paired $t$ tests showed that rats' performance in the low-memory-load condition was not significantly better than in the high-memory-load condition, $t(9)=-1.245, p=.878$, one-tailed. The $95 \%$ confidence interval was $2.365<\mu<$ 3.157 and $1.900<\mu<3.037$ for the low-memory-load and the high-memory-load conditions, respectively. Thus, although rats' performance in the both conditions was significantly better than the chance level of 3.5 , they were not significantly different from each other. The bottom panel of Fig. 8 shows the percentage correct of a first choice. According to 95\% confidence interval analysis, rats' performance was reliably better than chance $(16.67 \%)$ for both the low-memoryload condition $(19.261<\mu<37.406)$ and the high-memoryload condition $(18.518<\mu<38.149)$. Given that the mean percent correct was $28.333 \%$ for both the low-memory-load and the high-memory-load conditions, paired $t$ tests showed that the difference between the conditions was not significant, $t(9)=.000, p=.500$, one-tailed.

\section{Discussion}

Experiment 3 was designed to examine whether the significant directed forgetting effect in Experiment 2 reflected reallocation of memory resources from $\mathrm{F}$-items to $\mathrm{R}$-items. If rats could reallocate memory resources from F-items to R-items using R-cues and F-cues, better performance was predicted in the low-memory-load condition than in the high-memory-load condition. That is, rats could stop memory rehearsal for arms in which F-cues were presented and could reallocate memory resources from F-items to R-items in the low-memory-load 
(a) Low-memory-load condition

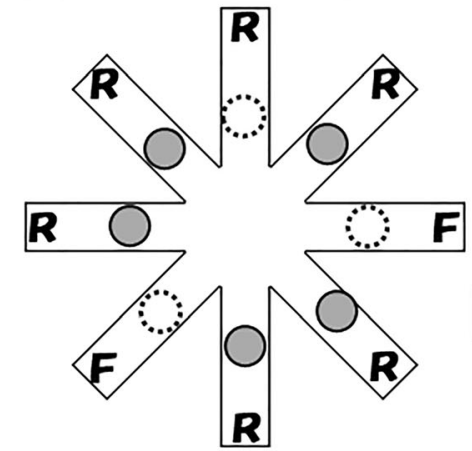

(1) Learning phase

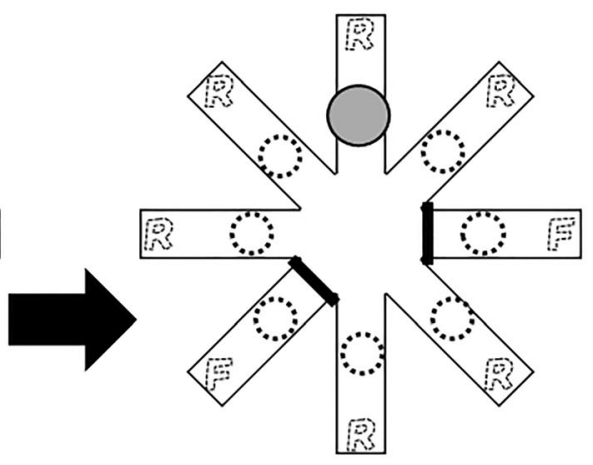

(2) Test phase

\section{(b) High-memory-load condition}

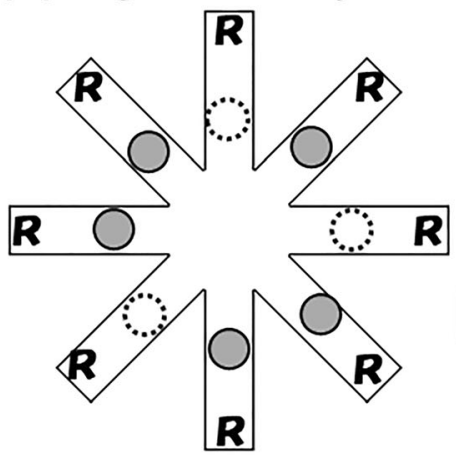

(1) Learning phase

Fig. 7 An overview of the procedure in Experiment 3. Rats received both low-memory-load trials (a) and high-memory-load trials (b) using a within-subjects design. Filled gray circles in the middle of the arms represent one food pellet in the learning phase (a1 and b1) or 20 food pellets in the test phase ( $\mathbf{a} 2$ and $\mathbf{b 2}$ ). Broken circles represent a nonreward in the middle food cups. Solid $\mathrm{R}$ and $\mathrm{F}$ at the end of the arms represent two qualitatively different foods presented as R- and F- cues in the end cup,

condition. In contrast, in the high-memory-load condition, since F-cues were not presented, rats had to remember during the learning phase all the arms that might be tested in the subsequent test phase. Contrary to our prediction, differences in both mean rank of correct responses and percent correct of first choice between these conditions was not statistically significant. Therefore, any positive evidence to support the idea of reallocation of memory resources from F-cues to R-cues was not evinced in Experiment 3.

The upper limits of the $95 \%$ confidential interval of mean rank of correct response and percentage correct of first choice were significantly better than chance, not only in the lowmemory-load condition but also the high-memory-load condition. Therefore, rats might not need to use F-cues if they were capable of remembering all eight arms. However, this explanation contradicts the results of Experiment 2, in which the same rats showed evidence of the use of F-cues. Apparently, the rats' performance deteriorated significantly

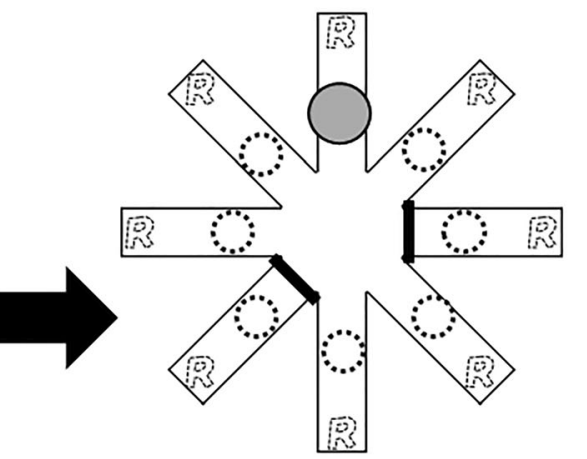

(2) Test phase

respectively. Broken $\mathrm{R}$ and $\mathrm{F}$ represent $\mathrm{R}$-cues and $\mathrm{F}$-cues presented in the prior learning phase. Bold lines at the entrances of the arms represent closed doors in the test phase ( $\mathbf{2} 2$ and $\mathbf{b 2}$ ). One lose-R arm was presented in the test of the low-memory-load condition (a), whereas one of three lose-R arms, selected randomly, was presented in the test of the highmemory-load condition (b)

when an F-arm was presented instead of an $\mathrm{R}$-arm in the probe tests of Experiment 2. These results strongly suggest that rats remembered arms selectively, using R-cues and F-cues from the learning phase for the subsequent test.

There were individual differences among rats in the decrement of performance in the probe test compared with the normal test in Experiment 2. Such individual differences may reflect differences in the levels of utilization of R-cues and F-cues to some extent. That is, greater deterioration in test performance in the probe test compared with the normal test would suggest greater utilization of R-cues and F-cues. This assumption predicts that rats that showed a greater directed forgetting effect in Experiment 2 might have better performance in the low-memory-load condition than in the highmemory-load condition in Experiment 3, given that the utilization of F-cues could reduce memory load in the lowmemory-load condition but not in the high-memory-load condition. 


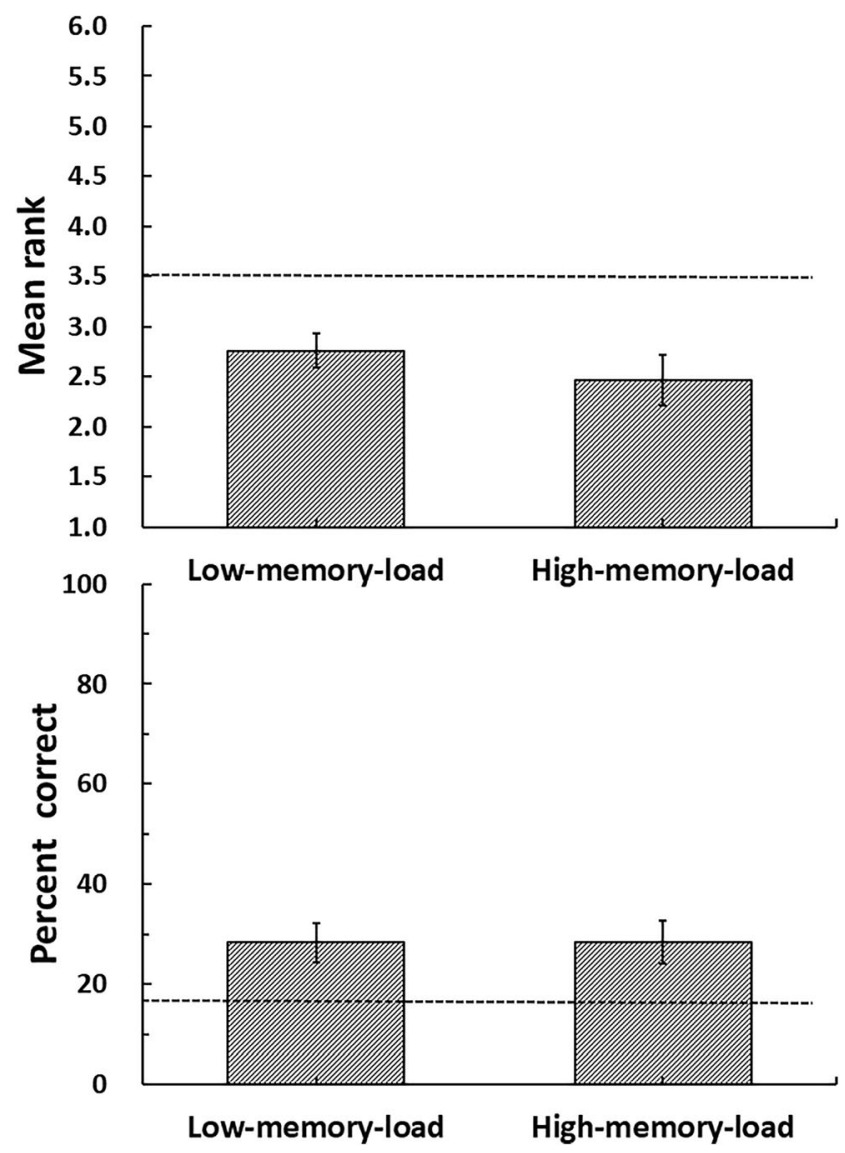

Fig. 8 Top panel: Mean rank of correct choice in the test phase of the lowmemory-load and high-memory-load conditions in Experiment 3. The broken line represents chance level (3.5). Bottom panel: Percentage correct of first choice in the test phase of the low-memory-load and the highmemory-load conditions. The broken line represents chance level $(16.67 \%)$. Error bars represent standard error of the mean

To test this hypothesis, the correlation between individual differences in directed forgetting effect in Experiment 2 and individual differences in performance between the lowmemory-load and high-memory-load conditions was analyzed. Two difference scores were calculated. First, the difference score was calculated by subtracting mean rank of the normal test from that of the probe test in Experiment 2 (see Fig. 6). In this difference score, a larger score means a greater directed forgetting effect in Experiment 2. The other difference score subtracted the mean rank of the low-memory-load condition from the high-memory-load condition (see Fig. 8) in Experiment 3. In this difference score, a larger score means better performance in the low-memory-load condition than in the high-memory-load condition. If rats that showed a greater directed forgetting effect in Experiment 2 had better performance in the low-memory-load condition than in the highmemory-load condition in Experiment 3, then a positive correlation between these two difference scores would be predicted. Contrary to the prediction, the correlation between these two difference scores was significantly negative $(r=-.660, p$
$=.038$, two-tailed). This negative correlation suggests that rats that evinced a greater directed forgetting effect - that is, rats that might utilize R-cues and F-cues more, showed poorer performance in the low-memory-load condition where Fcues could be utilized than in the high-memory-load condition where F-cues could not be utilized.

One possible explanation for this paradoxical result might be that while rats might exploit R-cues and F-cues to some extent to predict information in the subsequent test phase, such utilization of R-cues and F-cues might interfere with their main memory task. That is, the low-memory-load condition might actually be a "dual task" of memory, both of remembering the win or loss of a food pellet in the middle cup as well as the discrimination and selective processing task of R-cues and F-cues to predict use of the win/lose information in the subsequent test phase. Conversely, selective processing of Rcues and F-cues must not be needed in the high-memory-load condition since only R-cues were presented in this condition. Therefore, the analysis of the results of Experiments 2 and 3 at the individual level suggests that, although rats could use the direction of R-cues and F-cues to predict subsequent use of the win/lose information, a negative interference effect of using R-cues and F-cues might outweigh the benefit of reducing memory load by utilizing R-cues and F-cues. That is, although Experiment 3 failed to find results predicted from active control of memory resources, it might not necessary imply an absence of active control of memory processes in rats, and it suggests that processing R-cues and F-cues, in itself, is a discrimination task that requires memory resources in the present directed forgetting task.

If this were the case, we may need to devise an experimental condition in which the benefit of reducing the memory load by utilizing F-cues surpasses the processing load for R-cues and F-cues. One solution might be to use a radial maze with a larger number of arms-for example, a 12-arm maze. Increasing the number of to-be-forgotten F-arms would increase the benefit of reallocating memory resources from $\mathrm{F}$ arms to R-arms and might overcome the negative effect of processing R-cues and F-cues differentially. Thus, evidence of reallocation of memory resources might be shown.

\section{General discussion}

In Experiments 1 and 2 in the present study, compared with the normal test, in which the R-arm was presented in the test phase consistent with R-cuing and F-cuing in the learning phase, the rats' test performance significantly deteriorated in the probe test, in which, in contradiction to R-cuing and Fcuing in the learning phase, an F-arm was presented in the test phase instead of an R-arm. These results suggest that the directed forgetting task in the present study, in which R-items 
and F-items were presented in a same trial, was effective in examining directed forgetting in rats.

Previous studies of directed forgetting in rats had not properly controlled for nonmemorial artifacts, as Roper and Zentall (1993) pointed out. In contrast, as discussed in Experiment 1, the directed forgetting effect shown in the present study cannot be explained in terms of such nonmemorial artifacts, such as a conditional frustration, inattention, or surprise in the probe test trial, or incompatibility between the substitution response required following an F-cue in the training trial and the response required in the probe trial.

However, there may be some concern about the possibility that rats might have learned to avoid arms in the test phase where F-cues were presented in the prior learning phase. Consequently, arms in which F-cues were presented in the previous learning phase were simply excluded from the test phase by keeping their doors closed. Since rats never experienced any discrimination training with differential reinforcement - that is, rats received reinforcement for responses to the correct lose-R arm, but they never experienced nonreinforcement for a response to the incorrect lose-F arms - it is unlikely that rats learned to avoid the arms in which F-cues were presented in the learning phase through acquisition training. Moreover, if rats had learned to avoid arms in which F-cues were presented in the learning phase, it would be expected that test performance in the probe test would become poorer than the chance level. However, the mean ranks were 3.500 and 3.367 in Experiments 1 and 2, respectively, and they were not poorer than the chance level of 3.5. Additionally, the percentage correct of the rats' first choice in the probe test was $16.67 \%$ in both Experiments 1 and 2, which were equal to the chance level. These results of around, but not poorer than, chance performance, in the probe tests of Experiments 1 and 2, do not support the idea that rats learned to avoid F-arms in the test phase of the probe trial.

Another possible explanation might be that rats just learned to remember only a lose-R arm in the learning phase in which a large reward was presented in the subsequent test phase and that they did not learn anything about other of lose-F and win$\mathrm{R}$ arms in the learning phase at all. In this case, rats just learned to respond to a lose- $\mathrm{R}$ arm and responded to the other arms randomly in the test phase of a trial. This strategy might explain the around chance test performance in the probe tests in Experiments 1 and 2. The strategy might also explain almost equal test performance in the low-memory-load and the high-memory-load conditions in Experiment 3, if it could be assumed that rats could generalize the strategy to all the three lose- $\mathrm{R}$ arms presented in the high-memory-load condition and remember all of them as equally well as the one lose/ $\mathrm{R}$ arm in the low-memory-load condition.

However, this strategy cannot explain the reliable negative correlation between individual differences in directed forgetting effects in Experiment 2 and ones in performance in the low-memory-load and high-memory-load conditions in Experiment 3, mentioned in the Discussion of Experiment 3. The correlation suggests that as rats used R-cues and F-cues more, they showed poorer performance in the low-memoryload condition, in which F-cues were available, than in the high-memory-load condition, in which F-cues were not available. The strategy of remembering only a lose- $\mathrm{R}$ arm would explain individual differences in the directed forgetting effect in Experiment 2 in terms of the different degrees to which rats remembered only the lose-R arm using the R-cue or they remembered three lose arms (one lose- $\mathrm{R}$ and two lose-F arms) regardless of R-cues and F-cues in Experiment 2. In the former extreme case, it is expected that rats that learned to remember only one lose/ $\mathrm{R}$ arm would show equal (in case rats could remember one lose- $\mathrm{R}$ arm in the low-memory-load condition and the three lose- $\mathrm{R}$ arm in the high-memory-load condition equally) or better performance in the low-memory-loadcondition than in the high-memory-load condition because such rats had to remember only one lose-R arm in the lowmemory-load-condition in contrast to three lose- $\mathrm{R}$ arms in the high-memory-load condition in Experiment 3. However, it would be expected that if rats learned to respond to the three lose arms regardless of R-cues and F-cues, they would show equal performance in the low-memory-load and highmemory-load conditions because three lose arms (one lose- $\mathrm{R}$ and two lose-F arms in the low-memory-load condition and three lose- $\mathrm{R}$ arms in the high-memory-load condition) were equally presented in both conditions. Therefore, this analysis predicts only positive or no correlation between the degree of utilization of R-cues and F-cues in Experiment 2 and performance in the low-memory-load and the high-memory-load conditions in Experiment 3. Therefore, the significant negative correlation between these data would never have been predicted in terms of the strategy of remembering only lose- $\mathrm{R}$ arm in the learning phase and suggests that, generally speaking, rats learned something about both R-cues and Fcues. However, since individual rats may adopt different strategies and the present studies do not allow precise analysis of individual differences in learning strategy, the possibility of a simpler learning strategy should be considered carefully in future directed forgetting studies using the radial maze.

In these experiments, memory items were the win or loss of a food pellet presented in the middle cup and R-cues and Fcues were two qualitatively different foods presented in the end cups. Therefore, there is a possibility that rats discriminated arms in the test phase based on compound cues that consisted of a combination of reward events in the middle and end cups. Namely, the R-cues and F-cues in the end cups might not serve as independent cues, where a compound "lose+R" cue might serve as a positive stimulus, while the "win+R" cue might serve as a negative stimulus, and "lose+ F" cue signal exclusion from testing. Although the possibility that rats might learn to utilize reward events in the middle and 
end cups as compound cues is not in contradiction to the notion that rats in the present study controlled memory rehearsal based on R-cues and F-cues, it should be clarified that R-cues and F-cues can serve as independent cues. One promising way to examine whether R-cues and F-cues can serve as independent cues might be to test transfer of cue function from the original directed forgetting task to another task. Some previous studies reported the absence of transfer of R-cue and F-cue functions from original memory items to novel ones (e.g., Grant, 1988). However, Roper, Chaponis, and Blaisdell (2005) showed significant transfer of R-cue and F-cue functions between two different DMTS tasks in the pigeon. The possibility that R-cues and F-cues might serve as an independent cue or "higher order instruction" (Roper et al., 2005) in the present study could be tested by examining the transfer of the R-cues and F-cues among two or more directed forgetting tasks using different memory items and R-cues and F-cues.

Another possible problem in the present study might relate to the presentation of memory items (win or loss of a food pellet in the middle cup) and direction cues (R-cue or F-cue foods in the end cup) in the same maze arm. We assumed that rats start to remember an item when they encounter a win or loss in the middle cup and then differentially control rehearsal for the items after receiving R-cues or F-cues in the end cup. In this case, the directed forgetting procedure of the present study is involved in active control of rehearsal of memory items. However, the arm to-be-remembered or one to-be-forgotten must still be present when rats received R-cues or F-cues in the end cup. If rats combined win/lose information with arm information when they received R-cues or F-cues, the directed forgetting procedure in the present study might involve active control of encoding or memorization of arm information as well as rehearsal of memory items. Schwartz (1986, Experiment 2) presented a sample stimulus and a direction cue simultaneously to pigeons. Poorer test performance was observed in the probe test than in the normal test. Since the sample stimulus and R-cue or F-cue were presented simultaneously, the possibility that the results might reflect active control of encoding, but not rehearsal of sample stimulus, could not be excluded. Although the findings of the present study strongly suggest that rats showed active control of memory processes, at least of rehearsal or encoding, in order to elucidate rats' ability to control rehearsal specifically, reexamination with an improved procedure-for example, presenting R-cues and F-cues at the central platform after exiting an arm, would be needed.

Many studies have been conducted after Maki, Gillund, Hauge, and Siders (1977) first reported directed forgetting in pigeons using the omission procedure in the DMTS task. However, after the influential critical review of the initial directed forgetting studies by Roper and Zentall (1993), the number of studies of directed forgetting in nonhuman animals decreased, possibly because of the difficulty of controlling nonmemorial artifacts appropriately. More recently, however, some studies have investigated the neural activity of the prefrontal cortex in pigeon, using a directed forgetting paradigm with the omission procedure (e.g., Milmine, Rose, \& Colombo, 2008a; Milmine, Watanabe, \& Colombo, 2008b). These studies have found differential neural activity following R-cues or F-cues. But a subsequent study showed that the neural activities correlated with R-cues and F-cues were associated with the presence or absence of subsequent rewards, not the sample memory (Browning, Overmier, \& Colombo, 2011). These recent findings also suggest the importance of controlling nonmemorial artifacts in directed forgetting studies in nonhuman animals, as Roper and Zentall (1993) suggested.

The present study suggests that rats can actively control memory rehearsal or, at least, encoding. However, we were unable to obtain direct evidence of reallocation of memory resources from F-items to R-items. To clarify species differences and the evolutional origin of active control of working memory processes, a reexamination of directed forgetting in rats with a more finely discriminative task is necessary in order to tease apart these different aspects of memory.

\section{References}

Bjork, R. A. (1972). Theoretical implications of directed forgetting. In A. W. Melton \& E. Martin (Eds.), Coding processes in human memory (pp. 217-235). Washington, DC: V. H. Winston.

Brown, M. F. (1990). The effects of maze-arm length on performance in the radial-arm maze. Animal Learning \& Behavior, 18, 13-22. doi: https://doi.org/10.3758/BF03205234

Brown, M. F., \& Lesniak-Karpiak, K. B. (1993). Choice criterion effects in the radial-arm maze: Maze-arm incline and brightness. Learning and Motivation, 24, 23-39. doi:https://doi.org/10.1006/lmot.1993. 1002

Browning, R., Overmier, J. B., \& Colombo, M. (2011). Delay activity in avian prefrontal cortex: Sample code or reward code? European Journal of Neuroscience, 33, 726-735. doi:https://doi.org/10.1111/ j.1460-9568.2010.07540.x

Capaldi, E. J., \& Miller, D. J. (1988). Counting in rats: Its functional significance and the independent cognitive processes that constitute it. Journal of Experimental Psychology: Animal Behavior Processes, 14, 3-17. doi:https://doi.org/10.1037/0097-7403.14.1.3

Cohen, J. S., Reid, S., \& Chew, K. (1994). Effects of varying trial distribution, intra- and extramaze cues, and amount of reward on proactive interference in the radial maze. Animal Learning \& Behavior, 22, 134-142. doi:https://doi.org/10.3758/BF03199913

Cohen, J. S., Sturdy, C., \& Hicks, M. (1996). Intratrial proactive interference in rats' serial alternation performance in the radial maze. Animal Learning \& Behavior, 24, 300-309. doi:https://doi.org/10. 3758/BF03198978

Etkin, M., \& D'Amato, M. R. (1969). Delayed matching-to-sample and short-term memory in the capuchin monkey. Journal of Comparative and Physiological Psychology, 69, 544-549. doi: https://doi.org/10.1037/h0028209 
Golding, J. M., Long, D. L., \& MacLeod, C. M. (1994). You can’t always forget what you want: Directed forgetting of related words. Journal of Memory and Language, 33, 493-510. doi:https://doi.org/10. 1006/jmla.1994.1023

Grant, D. S. (1981). Stimulus control of information processing in pigeon short-term memory. Learning and Motivation, 12, 19-39. doi: https://doi.org/10.1016/0023-9690(81)90023-0

Grant, D. S. (1982). Stimulus control of information processing in rat short-term memory. Journal of Experimental Psychology: Animal Behavior Processes, 8, 154-164. doi:https://doi.org/10.1037/00977403.8.2.154

Grant, D. S. (1988). Directed forgetting in pigeons: Tests of transfer of cue effectiveness across samples from different dimensions. Learning and Motivation, 19, 122-141. doi:https://doi.org/10. 1016/0023-9690(88)90009-4

Grant, D. S., \& Barnet, R. C. (1991). Irrelevance of sample stimuli and directed forgetting in pigeons. Journal of the Experimental Analysis of Behavior, 55, 97-108. doi:https://doi.org/10.1901/jeab.1991.5597

Grant, D. S., \& Soldat, A. S. (1995). A postsample cue to forget does initiate an active forgetting process in pigeons. Journal of Experimental Psychology: Animal Behavior Processes, 21, 218228. doi:https://doi.org/10.1037/0097-7403.21.3.218

Kaiser, D. H., Sherburne, L. M., \& Zentall, T. R. (1997). Directed forgetting in pigeons resulting from the reallocation of memorymaintaining processes on forget-cue trials. Psychonomic Bulletin \& Review, 4, 559-565. doi:https://doi.org/10.3758/BF03214349

Maki, W. S., Gillund, G., Hauge, G., \& Siders, W. A. (1977). Matching to sample after extinction of observing responses. Journal of Experimental Psychology: Animal Behavior Processes, 3, 285296. doi:https://doi.org/10.1037/0097-7403.3.3.285

Maki, W. S., \& Hegvik, D. K. (1980). Directed forgetting in pigeons. Animal Learning \& Behavior, 8, 567-574. doi:https://doi.org/10. 3758/BF03197771

Maki, W. S., Olson, D., \& Rego, S. (1981). Directed forgetting in pigeons: Analysis of cue functions. Animal Learning \& Behavior, 9, 189-195. doi:https://doi.org/10.3758/BF03197819

Maxwell, S. E., Lau, M. Y., \& Howard, G. S. (2015). Is psychology suffering from a replication crisis? What does "failure to replicate" really mean? American Psychologist, 70, 487-498. doi:https://doi. org/10.1037/a0039400

Miller, W. S., \& Armus, H. L. (1999). Directed forgetting: Short-term memory or conditioned response? The Psychological Record, 49, 211-220. doi:https://doi.org/10.1007/BF03395317

Milmine, M., Rose, J., \& Colombo, M. (2008a). Sustained activation and executive control in the avian prefrontal cortex. Brain Research Bulletin, 76, 317-323. doi:https://doi.org/10.1016/j.brainresbull. 2008.02.009

Milmine, M., Watanabe, A., \& Colombo, M. (2008b). Neural correlates of directed forgetting in the avian prefrontal cortex. Behavioral Neuroscience, 122, 199-209. doi:https://doi.org/10.1037/07357044.122.1.199

Olton, D. S. (1978). Characteristics of spatial memory. In S. H. Hulse, H. Fowler, \& W. K. Honig (Eds.), Cognitive processes in animal behavior (pp. 341-373). Hillsdale, NJ: Erlbaum.

Parker, B. K., \& Glover, R. L. (1987). Event duration memory: The effects of delay-interval illumination and instructional cuing.
Animal Learning \& Behavior, 15, 241-248. doi:https://doi.org/10. 3758/BF03205018

Phelps, M. T., \& Roberts, W. A. (1991). Pattern tracking on the radial maze: Tracking multiple patterns at different spatial locations. Journal of Experimental Psychology: Animal Behavior Processes, 17, 411-422. doi:https://doi.org/10.1037/0097-7403.17.4.411

Roberts, W. A. (1972). Short-term memory in the pigeon: Effects of repetition and spacing. Journal of Experimental Psychology, 94, 74-83. doi:https://doi.org/10.1037/h0032796

Roberts, W. A., \& Dale, R. H. (1981). Remembrance of places lasts: Proactive inhibition and patterns of choice in rat spatial memory. Learning and Motivation, 12, 261-281. doi:https://doi.org/10. 1016/0023-9690(81)90009-6

Roberts, W. A., Mazmanian, D. S., \& Kraemer, P. J. (1984). Directed forgetting in monkeys. Animal Learning \& Behavior, 12, 29-40. doi:https://doi.org/10.3758/BF03199810

Roper, K. L., Chaponis, D. M., \& Blaisdell, A. P. (2005). Transfer of directed-forgetting cues across discrimination tasks with pigeons. Psychonomic Bulletin \& Review, 12, 1005-1010. doi:https://doi. org/10.3758/BF03206435

Roper, K. L., Kaiser, D. H., \& Zentall, T. R. (1995). True directed forgetting in pigeons may occur only when alternative working memory is required on forget-cue trials. Animal Learning \& Behavior, 23, 280 285. doi:https://doi.org/10.3758/BF03198924

Roper, K. L., \& Zentall, T. R. (1993). Directed forgetting in animals. Psychological Bulletin, 113, 513-532. doi:https://doi.org/10.1037/ 0033-2909.113.3.513

Santi, A., \& Savich, J. (1985). Directed forgetting effects in pigeons: Remember cues initiate rehearsal. Animal Learning \& Behavior, 13, 365-369. doi:https://doi.org/10.3758/BF03208011

Schwartz, B. (1986). Response stereotypy without automaticity: Not quite involuntary attention in the pigeon. Learning and Motivation, 17, 347-365. doi:https://doi.org/10.1016/00239690(86)90003-2

Stonebraker, T. B., \& Rilling, M. (1981). Control of delayed matching-tosample performance using directed forgetting techniques. Animal Learning \& Behavior, 9, 196-201. doi:https://doi.org/10.3758/ BF03197820

Stonebraker, T. B., Rilling, M., \& Kendrick, D. F. (1981). Time dependent effects of double cuing in directed forgetting. Animal Learning \& Behavior, 9, 385-394. doi:https://doi.org/10.3758/BF03197848

Tu, H.-W., \& Hampton, R. R. (2014). Control of working memory in rhesus monkeys (macaca mulatta). Journal of Experimental Psychology: Animal Learning and Cognition, 40, 467-476. doi: https://doi.org/10.1037/xan0000030

Zentall, T. R., Roper, K. L., \& Sherburne, L. M. (1995). Most directed forgetting in pigeons can be attributed to the absence of reinforcement on forget trials during training or to other procedural artifacts. Journal of the Experimental Analysis of Behavior, 63, 127-137. doi: https://doi.org/10.1901/jeab.1995.63-127

Publisher's note Springer Nature remains neutral with regard to jurisdictional claims in published maps and institutional affiliations. 